\title{
LE FANTASME DU DÉMIURGE : L'ARCHITECTE SOUMIS À LA TENTATION DU POUVOIR
}

\author{
Author(s) / Auteur(s) : \\ Damien CLAEYS \\ Architecte, Docteur en art de bâtir et urbanisme, Chargé de cours \\ Faculté d'architecture, d'ingénierie architecturale, d'urbanisme (UCL) \\ damien.claeys@uclouvain.be
}

\begin{abstract}
Résumé :
Confronté aux limites perceptives de la conscience, l'être humain développe pour agir une configuration augmentée, évolutive et opérationnelle du réel. Le décalage permanent entre les réactions parfois incompréhensibles du réel (incertitude) et la réalité patiemment construite par l'être humain (pseudo-certitude) est à la source d'une angoisse existentielle latente.

Pour permettre à chacun d'agir dans la vie de tous les jours, cette angoisse existentielle est transmise à d'autres en leurs donnant la responsabilité de configurer le réel augmenté à notre place.

Parmi d'autres, deux processus permettent à certains de configurer le réel augmenté à la place d'autres : la mise en place de systèmes politiques pour configurer le réel augmenté du peuple en jouant sur les interactions entre les membres du système socioculturel et la projetation/fabrication d'artefacts architecturaux pour configurer l'environnement écosystémique dans lequel habite le peuple.

Des rapports étroits existent entre les systèmes politiques et l'architecture puisque ces deux processus équilibrent, chacun à leur manière, les rapports dialogiques entre l'être (auto)organisé, le système socioculturel et l'environnement écosystémique.

Des jeux de pouvoir existent entre le gouvernant attiré par la figure du guide et l'architecte obsédé par le fantasme du démiurge.
\end{abstract}

\section{Keywords / Mots-clés :}

architecture, conception, pouvoir, démocratie, totalitarisme, finalité, système, réel, réalité, architecte, démiurge

\section{CONFIGURATION AUGMENTÉE DU RÉEL}

La quantité d'informations potentiellement perceptible depuis le réel dépasse les capacités cognitives $\mathrm{du}$ cerveau humain. L'être humain souffre d'une "bounded rationality" (Simon, 1957) qui rend impossible l'appréhension totale du réel par la conscience ${ }^{1}$. À travers le filtre cognitif de ses organes perceptifs, l'être humain n'interprète qu'une partie limitée des informations disponibles dans le réel. À défaut d'une connaissance complète du réel $R$, il construit une réalité $R^{\prime}$, appelée ici le "réel augmenté" (Claeys, 2013) ${ }^{2}$.

\footnotetext{
1 La "rationalité bornée" est utile puisque "tous les organismes humains vivent dans un environnement qui génère des millions de bits d'informations nouvelles chaque seconde, mais le goulot d'étranglement de l'appareil perceptif n'admet pas plus de 1.000 bits par seconde, et probablement beaucoup moins" (Simon 1959).

2 Le réel augmenté $\left(R^{+}\right)$est "un double du réel co-construit par l'homme - qualifié d'augmenté - contre lequel, impertinent, le réel résiste dès qu'il est provoqué. Il possède plusieurs partitions - qui ne font pas partie du réel - tels que les réalités perçue, conçue, vécue, mythique, objective, subjective, intersubjective... Il se confronte constamment au réel $(R)$ qui reste un au-delà qui lui échappe. Le réel augmenté est à distinguer de la réalité augmentée" (Claeys, 2013). Contrairement à la réalité augmentée qui nécessite des moyens techniques pour surimposer des informations au réel perçu, le réel augmenté est produit directement par notre conscience. Le réel augmenté est structuré par plusieurs partitions correspondantes à des réalités différentes : les réalités intersubjective, subjective et objective. Dans mes précédents travaux j'utilisais un plus pour distinguer $R^{+}$de $R$. Mais le réel augmenté est, à la fois, moins que le réel puisque notre conscience souffre d'une limite cognitive dans le traitement des informations extérieures et plus que le réel puisque notre conscience est capable de faire émerger un monde intérieur qui n'existe pas dans le réel. Dorénavant, je propose donc d'utiliser un prime pour distinguer le réel augmenté du réel.
} 
Une boucle de rétroaction est à l'œuvre entre les effets du réel sur le corps et les actions du corps sur le réel. L'être humain est donc un être (auto)organisé en interaction dynamique avec son environnement qui adapte son comportement en fonction du projet téléologique - finalité projective interne - qu'il poursuit à court/moyen/long terme ${ }^{3}$. L'être peut donc être considéré comme un agens (agissant) participe présent du verbe latin ago (agir) - dans le sens où cet être (auto)organisé est une entité qui agit, qui opère d'abord en fonction d'un projet purement personnel, mais qui peut également être chargée/investie d'une mission pour d'autres.

L'être humain configure patiemment un deuxième monde - le réel augmenté - pour calmer son angoisse existentielle en projetant sur un réel (source d'incertitude) un ordre (inter)subjectif pour assoir des (pseudo-)certitudes. À côté du $R$, il développe patiemment un $R^{\prime}$ en confrontant son (auto)référentiel avec les informations issues de ses interactions répétées avec l'environnement, mais également avec les autres membres du groupe socioculturel auquel il appartient. Ces interactions sont donc tributaires de l'action structurante des systèmes politiques qui régissent les interactions entre les membres du groupe et des limites de la scène spatialisée dans lesquelles elles ont lieu. Ce second monde fait la richesse de l'espèce humaine puisqu'il permet l'émergence de l'art, de la science, de la religion... mais également le malheur de celle-ci puisqu'il fournit un support souple à toutes les angoisses existentielle de l'être humain confronté à un réel résistant. De cette distinction fondamentale entre $R$ et $R^{\prime}$, l'être humain a le besoin constant d'organiser le monde qui l'entoure, de (re)configurer son image du réel pour calmer ses angoisses existentielles.

La stabilité du $R^{\prime}$ est régulièrement remise en jeu lorsque le $R$ résiste à nos projections (inter)subjectives. Nos tentatives de confrontations directes au réel sont vouées à l'échec. Seule demeure l'accumulation des informations que nous tirons de nos essais/erreurs et la vérification par l'expérience qui contredit régulièrement la conception fantasmée du réel que nous avons. Aucune correspondance complète n'est possible entre le réel et nos réalités multiples. Une différence existe donc entre $R$ et $R^{\prime}$, un solde générateur d'angoisse existentielle et d'incertitude sur lequel la structure logique patiemment élaborée de $R^{\prime}$ n'a pas prise. Ce solde fait virtuellement partie du non-réel augmenté $\neg R^{\prime}$, constitué de l'infinité de tous les possibles de l'imaginaire, non-activés dans $R^{\prime}$. L'être humain peine à appréhender cet écart négatif (limite perceptive) et positif (imagination débridée) dont les contours évoluent constamment. Pour protéger son assise existentielle et stabiliser les contours de $R^{\prime}$, il a inévitablement recours à des subterfuges. Et dans toute société structurée, l'instrumentalisation de subterfuges communs aux membres du groupe est déléguée à d'autres.

Dès les sociétés archaïques, l'être humain façonne des réalités multiples et complexes avec lesquelles il pense appréhender - voire maîtriser - le réel. Il déplie (analyse) et replie (synthèse) constamment l'image construite de ce dernier pour tenter de le connaître. Il est aux prises avec un $R$ sur lequel il projette le visible et l'invisible. D'un côté, un monde ordonné selon la conception du monde du groupe social auquel il appartient. De l'autre, un monde parallèle contenant des entités surnaturelles bénéfiques ou maléfiques (dieux, âmes des morts, spectres...). Les deux mondes sont entremêlés. Ces deux constituants du $R^{\prime}$, le visible et l'invisible, s'enchevêtrent et leurs contours sont variables. Il utilise des subterfuges pour donner accès au monde invisible qui est difficilement descriptible à partir d'observations rationnelles (l'art, le mythe, le rite, l'artisanat...).

Aujourd'hui, les entités surnaturelles qui habitent l'invisible sont plutôt associées aux agents technoéconomiques et/ou politiques (Couloubaristsis, 2014). Sans recours à la magie et/ou à la religion, les phénomènes observés s'expliquent plutôt par simplification à l'aide de "systèmes symboliques descriptifs" tels que la science et la philosophie (Norberg-Schulz, 1974). Malgré ces efforts répétés de "désenchantement du monde" (Weber, 1905), l'enchevêtrement de ces deux mondes est encore largement présent dans notre société contemporaine à l'aide de "systèmes symboliques nondescriptifs" tels que l'art et la religion (Norberg-Schulz, 1974).

\footnotetext{
3 Un projet téléologique tel que décrit ici est le catalyseur d'un processus d'action menant à une finalité interne (celle fixée par le système lui-même). Ce type de projet est à distinguer d'une programmation téléonomique associée à une finalité externe (celle du modélisateur du système) et d'une destinée théologique sous l'effet d'une cause extérieure (celle d'une intelligence divine).
} 
Parmi d'autres, deux moyens de (re)configurer le réel pour d'autres interagissent fréquemment : la politique et l'architecture.

Tous les êtres humains sont des organisateurs du $R^{\prime}$ en puissance. Parmi eux, légitimes ou non, les gouvernants prennent la responsabilité de le faire pour d'autres : les gouvernés. La configuration du $R^{\prime}$ par d'autres est à l'origine des systèmes politiques et, parmi ces derniers, de la démocratie. Les gouvernés acquièrent donc une liberté d'action relative supportée par une insouciance relative pendant que les gouvernants organisent le $R^{\prime}$ pour eux, en partie ou en tout. En général, l'environnement construit est la scène globale des interactions socioculturelles organisées par le système politique au pouvoir. En particulier, l'achitecture assure à l'agens une situation existentielle locale dans le monde, un point $p$ dans le $R^{\prime}$, un subterfuge pour calmer l'angoisse existentielle inhérente à la condition humaine. Les gouvernants utilisent régulièrement l'environnement construit en général et l'architecture en particulier pour influer sur les interactions socioculturelles et l'assise existentielle des gouvernés. Dans ce jeu d'interactions qui le dépasse, l'architecte possède un statut professionnel ambiguë entre les gouvernants desquels dépendent les commandes et les gouvernés auxquels il fournit des habitats.

\section{POTENTIEL, POUVOIR, PROJET}

À partir de l'interaction entre un sujet et des décisions prises par d'autres, le philosophe français Michel Foucault (1984) définit l' "exercice du pouvoir" comme "un ensemble d'actions sur des actions possibles". Selon Foucault, "il opère sur le comportement de sujets agissants : il incite, il induit, il détourne, il facilite ou il rend plus difficile ; à la limite, il contraint ou empêche absolument". Mais cet exercice "est bien toujours une manière d'agir sur un ou sur des sujets agissants, et ce tant qu'ils agissent ou qu'ils sont susceptibles d'agir. Une action sur des actions."

Le verbe pouvoir vient du verbe latin providere (voir en avant, organiser d'avance). Le pouvoir d'un agent correspond donc aux actions potentielles qu'il peut mener en fonction d'un projet, d'une anticipation pour organiser le réel d'autres agents. Le verbe pouvoir signifie avoir la capacité ou avoir la possibilité de faire quelque chose. Avoir du pouvoir, c'est avoir le potentiel de faire et de vouloir, c'est-à-dire un potentiel objectif d'action en fonction d'un projet (inter)subjectif. Avoir du pouvoir, c'est également pourvoir aux besoins de l'autre. Être au pouvoir équivaut à avoir - il faut l'espérer - le projet politique de faire quelque chose, sans avoir nécessairement la capacité de le faire. La visée téléologique de l'agent et les processus opérationnels qui permettent de la mettre en œuvre agissent à des niveaux logiques différents.

Un parallélisme évident apparaît alors entre l'exercice du pouvoir et l'activité de projetation des architectes. Le mot projeter est lié aux mots latins por (en avant), jacere (jeter) et objicere (jeter en avant). La conception d'un projet d'architecture est justement la capacité de voir en avant en fonction d'une visée téléologique, d'organiser d'avance le $R^{\prime}$ d'autres agents pour pourvoir à leurs besoins, sans avoir nécessairement le pouvoir de réaliser l'édifice.

Le pouvoir s'exerce d'abord dans les limites des contraintes du réel, mais il s'exerce également dans les limites du $R^{\prime}$ projeté sur le $R$. Les limites à la réalisation du projet résultent des interactions de l'agent avec d'autres agents ou avec des contraintes de l'environnement. Ayant chacun un potentiel d'action en fonction d'un projet personnel, deux agents ne peuvent réaliser - rendre réel - une partie de ce potentiel que si leurs actions sont compatibles.

L'exercice du pouvoir est donc principalement l'aptitude à gouverner. Le verbe gouverner vient du grec kubernesis (action de manœuvrer un bateau, conduire, naviguer mais aussi guider ou aider), composé de náô (naviguer) et kubaía (navire), menant au latin gubernare (administrer, diriger, conduire). Gouverner signifie l'action de diriger avec un gouvernail, ce qui signifie, par extension, l'action de diriger la conduite des choses/personnes et de leurs apporter des soins pour les maintenir en bon état, pour qu'elles ne périssent pas ${ }^{4}$. Autrement dit, gouverner, c'est orienter et maintenir un

\footnotetext{
${ }^{4}$ Le mot cybernétique vient également du grec kubernesis puisqu'il s'agit de la science du contrôle et de la communication chez l'animal et la machine (Wiener, 1948). Pour le mathématicien français Louis Couffignal (1963), la cybernétique est "l'art et la science d'assurer l'efficacité de l'action". Et mesurer l'efficacité d'une action, c'est voir si l'action mène à un but prédéfini, une finalité. Des liens étroits existent donc entre la question du pouvoir et les origines de la cybernétique des
} 
système politique en fonction d'un projet. Ce système est le produit des interactions répétées entre les membres d'un groupe socioculturel.

L'exercice du pouvoir est la capacité d'action d'un gouvernant à gérer - en fonction d'un projet - le jeu des relations de pouvoir entre les différents agents d'un système socioculturel. Pour Foucault (1984), l'exercice du pouvoir consiste à "conduire des conduites" et à "aménager la probabilité". En effet, le gouvernant conduit les gouvernés, alors que les gouvernés se conduisent. Ces derniers acquièrent une manière de se comporter dans "un champ plus ou moins ouvert de possibilités". Le mode d'action de gouverner consiste à "structurer le champ d'action éventuel des autres". Chaque membre du système socioculturel poursuit des (méta)finalités différentes associées à un potentiel d'actions différentes, ayant la probabilité d'être réalisées. Des gouvernants organisent un $R^{\prime}$ commun pour des gouvernés qui ne partagent au départ pas le même $R^{\prime}$.

Pour réguler les réalisations des potentiels des différents membres d'un groupe, des règles émergent progressivement du système socioculturel. En fonction d'un projet d'intérêt général, les gouvernants codifient les interactions des gouvernés et créent des institutions pour développer les modalités opérationnelles d'application et d'actualisation de ces règles. Une relation éthique bidirectionnelle s'installe entre gouvernants et gouvernés dans l'élaboration des règles qui fixent les limites des potentiels d'actions de chacun. D'un côté, tout gouvernant assume ses promesses - le mot latin sponsio (promesse) donne le verbe respondere (se porter garant, répondre de) - lorsqu'il agit pour d'autres. Tout gouvernant a la responsabilité, le devoir de répondre de ses actions. De l'autre, tout gouverné accepte, jusqu'à une limite donnée, d'être déresponsabilisé. Cette déresponsabilisation est proportionnelle à l'insouciance relative de l'agent à donner le pouvoir à d'autres.

\section{PROXIMITÉ TRIALOGIQUE \\ ENTRE ARCHITECTURE ET SYSTÈME POLITIQUE}

Selon le théoricien de l'architecture norvégien Christian Norberg-Schulz (1974), les édifices sont des médias institués et porteurs potentiels de significations existentielles dont l'être humain peut faire l'expérience. Les artefacts architecturaux concrétisent un ensemble de significations articulées. Mis en relation, ils forment un véritable "système symbolique". Les architectures sont alors capables d'assurer, en partie, la formation de l' "assise existentielle" chez l'habitant en lui permettant d'expérimenter un "environnement signifiant" à la fois avec lui-même, avec son environnement et avec les autres membres du groupe social dont il fait partie. Le fait de "concourir à rendre l'existence humaine signifiante" est, selon lui, "le véritable but de l'architecture". Toutes les autres fonctions des architectures pour parer aux besoins purement physiques de l'homme "peuvent être satisfaites sans l'architecture". Autrement dit, parmi d'autres moyens possibles, les édifices sont des médias capables d'aider l'être humain à partager partiellement un $R^{\prime}$ avec d'autres au sein d'un environnement construit. L'environnement construit dans le $R$ est le support d'un potentiel de projections symboliques dans le $R^{\prime}$.

L'architecture divise l'étendue en créant un dedans (pseudo-certitude) par rapport à un dehors (incertitude) pour que l'être humain puisse y trouver une assise existentielle. Elle crée un système relativement fermé pour protéger l'agens du système socioculturel et de l'environnement écosystémique qui l'entourent. Elle crée un nouvel écosystème, un ordre dans le désordre apparent du monde.

L'agens a le besoin fondamental de se situer existentiellement et donc d'organiser efficacement le $R$. Il donne alors procuration à d'autres - des politiques - pour organiser globalement l'environnement construit.

De ce qui précède, trois termes liés émergent : l'autre, l'être et l'environnement. En partant du principe qu'un architecte possède la métafinalité projective de créer des lieux, le travail de l'architecte est

systèmes fermés contrôlés par un pouvoir central qui assure une régulation homéostatique simple. La pensée systémique, dont la cybernétique est l'un des courants précurseurs, a historiquement pris ses distances avec l'étude des systèmes fermés en considérant les systèmes ouverts à l'aide de concepts dynamiques : auto-organisation, émergence, incertitude... 
d'équilibrer "les rapports trialogiques entre le système socioculturel, l'être (auto)organisé et l'environnement écosystémique" (Claeys 2013).

Rechercher cet équilibre en construisant des édifices, c'est agir sur le $R$ en configurant indirectement le $R^{\prime}$ d'autres personnes. Par la métafinalité qu'il poursuit, l'architecte se donne le pouvoir virtuel de configurer principalement l'environnement écosystémique par la projétation d'artefacts architecturaux dans le $R^{\prime}$ qui - par les relations qui les lient une fois construites dans le $R$-influencent indirectement le système socioculturel (scène spatialisée des interactions entre les membres du système socioculturel) et l'être (auto)organisé (espace privé de l'habitation).

Rechercher cet équilibre en mettant en place un système politique, c'est donner à des gouvernants le pouvoir virtuel, de configurer principalement le système socioculturel en fonction d'un projet dans le $R^{\prime}$ par l'organisation des relations entre les gouvernés en développant un état de droits (par exemple, une démocratie) dans le $R$ qui - par les interactions qu'ils entretiennent - influence indirectement la construction de l'être (auto)organisé et l'environnement écosystémique.

Entre l'architecture et le pouvoir politique, des liens réguliers apparaissent puisqu'ils peuvent se servir l'un de l'autre pour acquérir/légitimer le pouvoir d'agir sur l'environnement construit ou sur le fonctionnement de la société pour, enfin, agir sur les êtres (auto)organisés.

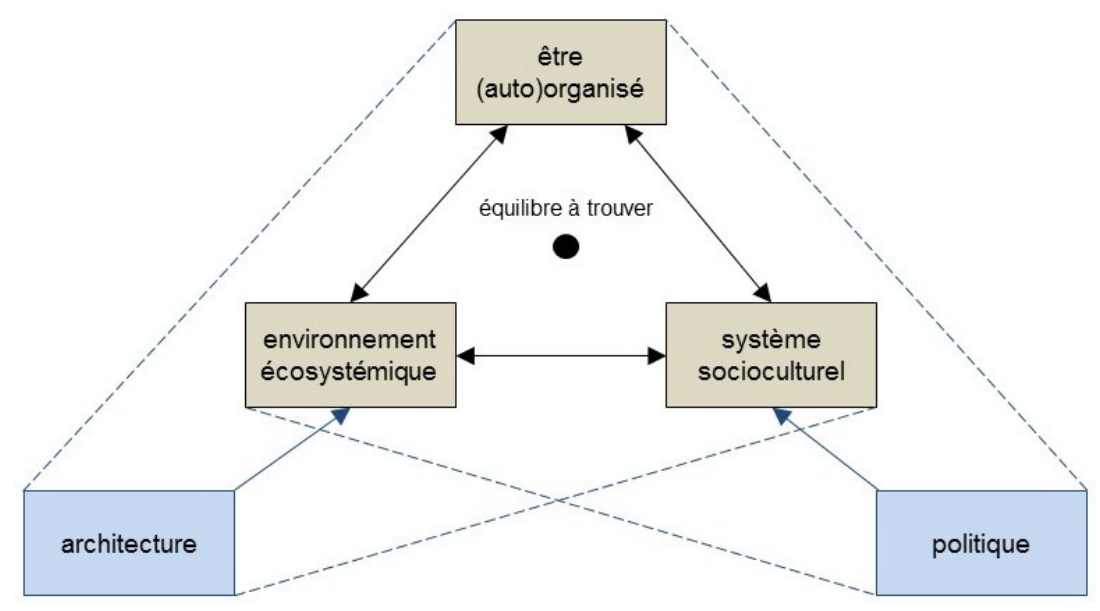

Figure 1. Proximité trialogique entre architecture et politique.

Les membres d'une société sont toujours en interaction dynamique entre eux, mais également avec l'environnement qui les entoure. Une grande partie de cet environnement est concrétisé à l'aide d'éléments architectoniques tels que des édifices : cet environnement construit est une production humaine - conçue avec ou sans architectes - liée à la conception du monde partagée par les membres de la société, ou du moins par les membres ayant le pouvoir d'organiser la société.

À la naissance, chaque nouveau membre d'une société se développe dans un déjà-là préconstitué, associé notamment à un environnement construit. Des boucles de rétroaction dynamiques apparaissent alors entre les trois pôles d'une dialogique : le système social, l'habitant et l'édifice. La société forme des acteurs qui matérialisent l'environnement construit, tandis que ce dernier conditionne le développement des membres de la société qui y vivent (et donc, parmi eux, d'autres acteurs potentiels qui, à leur tour, changeront la société et produiront de nouveaux artefacts architecturaux). Ce phénomène reste vrai quel que soit le type de système politique au pouvoir.

En modifiant l'environnement construit, les gouvernants laissent régulièrement des traces pérennes et durables dans le $R$ par la réalisation d'édifices à la monumentalité particulière, légués aux générations futures, leurs permettant un accès à la postérité. Au moyen d'édifices rappelant en permanence aux membres de la société - mais souvent aussi aux membres d'autres sociétés - la présence et le force d'un pouvoir (démocratique ou dictatorial), les gouvernants imposent progressivement une idéologie 
dans le $R^{\prime}$ des gouvernés. Lorsqu'un changement de pouvoir survient, ces édifices deviennent des cicatrices douloureuses (abandon, haine, honte...) qu'un usage ordinaire (oubli) ou une muséification (mythe) peut banaliser avec le temps.

Dans cette trialogique, c'est le pôle de l'être humain qui est le plus faible par rapport au système social et à l'écosystème. La durée de vie et l'influence d'un agens reste limitée par rapport aux deux autres pôles qui lui précèdent et lui succèdent. Pourtant l'être (auto)organisé est le seul à avoir le besoin existentiel d'occuper une situation dans le monde, dans laquelle des règles régissent les jeux de pouvoir à l'œuvre dans les interactions qu'il a avec d'autres être (auto)organisés ou avec l'environnement écosystémique. Les politiques font régulièrement référence à ce type de besoin pour manipuler l'opinion publique, affirmer ou pérenniser leur pouvoir.

\section{LE MYTHE DU POUVOIR DE L'ARCHITECTE}

Étymologiquement, l'architecture et le pouvoir politique sont liés depuis l'Antiquité.

Le mot politique vient du latin politice et du grec politikê, composé de polis (cité) et de tekhnê (art, habilité). Dès le Moyen Âge, l'italien Brunetto Latini (1265) pense que la politique est donc littéralement la "science du gouvernement des états", ce qui, pour le français Nicole Oresme (1370), "a rapport aux affaires publiques de l'état". Le mot police vient du grec politeia (l'art, la technique de/pour gouverner la cité). Le mot cité vient de polis, mais aussi du latin civitas (ensemble de citoyens, puis territoire où ils vivent et enfin ville). Le mot politique n'est utilisé dans le sens actuel que depuis le XIII ${ }^{\mathrm{e}}$ siècle et conserve donc étymologiquement des références à l'art de gérer l'environnement construit qui nous entoure. Au final, la politique est l'ensemble des pratiques, des faits, des institutions et des décisions d'un gouvernement, d'un état ou d'une société.

Le mot architecte vient du grec architektón (celui qui commande les ouvriers, celui qui dirige les travaux), composé des mots arkhein (commander) ${ }^{5}$ et tektôn (ouvrier, ouvrier travaillant le bois, ouvrier charpentier ou maçon) qui provient, avec le mot tékhnê (habileté, art), de l'indo-européen texo (travailler le bois ou le tissu, assembler), à l'origine du mot latin textus (tissé, tissu, trame, texte) ${ }^{6}$.

Au-delà d'une proximité étymologique (et en partie sémantique), l'architecture et le pouvoir politique sont en relation constante. Historiquement, l'architecte se place presque toujours du côté du pouvoir pour légitimer un statut professionnel souvent fragile et parce que seul un maitre de l'ouvrage puissant et riche peut lui commander la construction d'un édifice monumental.

Pour le critique d'architecture français Michel Ragon (1977), l'histoire de l'architecture "convie à la contemplation passive du chef-d'œuvre". Les seules références (re)connues de l'histoire de l'architecture sont les temples et les palais pour/par la classe dirigeante. L'habitat ordinaire est peu considéré par rapport aux pyramides, au Parthénon, au Panthéon, aux cathédrales ou au château de Versailles. Ainsi, quand des révolutions éclatent, les gouvernés qui n'ont pas accès à l'architecture commencent par brûler les architectures des gouvernants. L'architecture est alors le symbole du pouvoir politique en place : il faut détruire la première pour renverser le second.

À l'Antiquité, les structures spatiales traditionnelles sont hautement hiérarchisées, polarisées et codifiées et elles supportent directement la structure des interactions sociales de membres du groupe. Dans ce totalitarisme élémentaire, la survie du groupe socioculturel est la priorité. L'individu compte peu, son rôle est planifié à l'avance et sa liberté est relative. Ainsi, en Mésopotamie et en Égypte, les prêtres auraient pratiqué l'art de la divination pour diriger la construction des monuments. Pendant qu'ils dormaient, les dieux leurs soufflaient à l'oreille les plans des édifices. Les mêmes schèmes spatialisés se retrouvent partout et ils montrent les relations entre l'environnement construit et

\footnotetext{
5 Le préfixe grec archi- indique donc la hiérarchie en référence aux mots grecs arkhè (le principe, le fondement, ce qui vient en premier) et arkhi- (chef de), préfixe venant du verbe árkhô (commencer, mener, gouverner).

${ }^{6}$ Le mot architecte a une origine très ancienne puisque le mot tekton est déjà utilisé pour désigner un charpentier de marine ou de maison dans l'Iliade et dans l'Odyssée du poète grec Homère (fin du VIII ${ }^{\mathrm{e}} \mathrm{s}$. av. J.-C.) et architekton est utilisé pour désigner un homme de l'art qui commande aux ouvriers, architecte ou ingénieur, concepteur et réalisateur d'une œuvre exceptionnelle dans les Histoires de l'historien grec Hérodote (vers v ${ }^{\mathrm{e}}$ s. av. J.-C.).
} 
l'organisation politique du pouvoir en place (village bororo, polis grecque, ville étrusque, cité romaine...).

Au Moyen Âge, l'architecture est un ars serviles. Les édifices magnifient et imposent à tous la présence quotidienne du pouvoir temporel des rois et/ou du pouvoir spirituel de l'Église. Mais le magister operis (maître d'œuvre) de ces monuments qui doivent inspirer la crainte, le respect ou l'amour du pouvoir en place est souvent anonyme.

À la Renaissance, l'architecture n'est plus un ars serviles mais un ars liberalis, une matière fondée sur un savoir intellectuel ${ }^{7}$. L'architecte se bat pour acquérir une nouvelle figure sociale radicalement différente de celle des travailleurs manuels. Il devient fonctionnaire, courtisan ou protégé de grands mécènes. L'architecte devient un professionnel capable de définir de façon autonome les règles de son activité. Selon la nouvelle image qu'il donne de lui, l'architecte est savant (géomètre et ingénieur) et humaniste (nourri de la tradition antique qu'il commente et critique). Au début du XVI siècle, cette image devient sublime, l'architecte est à la fois un génie, créateur, artiste, démiurge, savant, ingénieur, intellectuel... Il est socialement déifié en tant qu'homme du dessein et du dessin. Placé à la tête du processus de construction, il ordonne le travail des corporations depuis le point de vue de la perspective et du plan. Ainsi, Michel-Ange est considéré comme un artiste divin, à la fois sculpteur, peintre et architecte.

Dans les écrits alchimiques du Moyen Âge ou ésotériques de la fin de la Renaissance, à côté d'illustrations plus abstraites (cercles, carrés, triangles), des représentations architecturées apparaissent progressivement (palais, citadelles, temples...). Elles représentent des processus irreprésentables (évolution du cosmos, création de la matière, monde de Dieu...). Les codes utilisés par ces modèles traduisent une vision hautement hiérarchisée, codifiée et précise du monde. Considérés comme parfaits, ces schèmes spatialisés organisent le monde des hommes pour leurs permettre d'atteindre automatiquement le bonheur. Fortement influencés par ces représentations, les architectes de l'époque projettent alors de nombreuses cités idéales pour le bien de la société en oubliant la liberté individuelle des habitants.

$\mathrm{Au} \mathrm{XVII}{ }^{\mathrm{e}}$ siècle, les architectes pactisent à nouveau avec le pouvoir en place pour conforter leur statut professionnel. L'Académie d'architecture est créée par Louis XIV en 1671. La noblesse de l'art des architectes doit les distinguer de simples artisans puisqu'ils connaissent "mille choses que l'on n'apprend point dans les conditions d'un simple artisan" (Perrault, 1673). L'architecte est un intellectuel qui dispose de règles savantes et esthétiques. L'académicien en tire un statut privilégié puisqu'il est évidement le seul à les comprendre. Ce système est rendu possible par l'autorité de l'Académie qui valorise le régime en place en se portant garante: du respect d'une théorie architecturale unique; de l'attribution de commandes officielles et de charges d'enseignements aux architectes académiciens. Les gens ordinaires n'ont pas droit à l'architecture pour eux-mêmes, puisqu'elle est du côté du pouvoir monarchique.

À partir du XVIII ${ }^{\mathrm{e}}$ siècle, la démocratie "apparaît en songe" (Ragon, 1977) plusieurs fois aux architectes. Issues de bonnes intentions rapidement détournées par le pouvoir en place, quatre tentatives marquantes d'intégration d'une dimension démocratique en architecture peuvent être relevées.

Après la Révolution française, les gouvernants ne sont plus des rois ou des religieux. Les architectes conçoivent des monuments en l'honneur de la démocratie ${ }^{8}$. Un grand nombre de ces projets sont restés

\footnotetext{
${ }^{7} \mathrm{Au}$ départ, sept ars liberalis (arts libéraux/nobles) sont définis dans le programme d'étude de la méthode scolastique médiévale constituée du trivium ou les arts de la langue (grammaire, dialectique, rhétorique) et du quadrivium ou les arts des mathématiques (arithmétique, musique, géométrie, astronomie). Selon l'église catholique, ces sept matières sont intellectuelles et intangibles, elles visent une connaissance désintéressée et supérieure. Les sept ars liberalis s'opposent aux sept ars serviles (arts serviles/mécaniques) qui concernent tous les savoir-faire et techniques utiles à la transformation, l'assemblage ou la mise en forme de la matière (fabrication de la laine, armement, navigation, agriculture, chasse, médecine, théâtre). En latin, au-delà d'une dimension esthétique, le mot ars signifie plutôt habilité, métier, connaissance technique.

${ }^{8}$ Cette période historique marque le passage d'une monarchie absolue à une république démocratique, elle est mouvementée du point de vue politique et elle serait sans doute aujourd'hui partiellement comparée à un régime totalitaire !
} 
sur le papier, faute de moyens pour les mettre en œuvre. De bonne foi, les architectes soutiennent la démocratie une première fois. Ils s'efforcent de faire le bonheur des hommes, mais ils les forcent à être heureux. En fait, ils imaginent une architecture idéale pour un homme tout aussi idéal. Une fois de plus, ils en oublient l'homme ordinaire.

$\mathrm{Au} \mathrm{XIX}{ }^{\mathrm{e}}$ siècle, l'image idéalisée de l'architecte créateur s'étiole parallèlement à l'émergence du capitalisme. Cette société moderne est confrontée aux effets de l'industrialisation naissante (grandes concentrations urbaines, exode rural, pollution et insalubrité...). Les pouvoirs politique et religieux s'affaiblissent parallèlement à l'enrichissement de la bourgeoisie qui préfère faire confiance à l'homme de science plutôt qu'à l'homme de l'art. L'ingénieur utilise les matériaux de son époque et réalise des défis techniques. Il s'impose progressivement face à l'architecte des beaux-arts, nostalgique d'un passé glorieux. L'idéologie progressiste dirigiste propose de faire le bonheur des hommes grâce à la science et à la technique, le culte de l'individu se développe et le groupe socioculturel éclate. En utilisant les compétences des ingénieurs, les architectes pensent soutenir une seconde fois la démocratie lorsqu'ils élèvent des monuments qui valorisent la marche universelle du progrès scientifique et technique (palais des machines, halls des expositions universelles, gares de chemins de fer, bourses de commerce...). S'ils ne construisent plus pour les rois et/ou l'Église, ils deviennent les pantins des bourgeois, nouveaux riches, pour lesquels ils construisent des ossatures modernes recouvertes de pastiches néoclassiques. Au début du $\mathrm{XX}^{\mathrm{e}}$ siècle, les architectes constitueront par ailleurs des ordres. Ce corporatisme passéiste est le produit de la mélancolie reconquérante du statut professionnel de l'architecte académicien. Les ordres interdisent à qui n'a pas le diplôme d'architecte d'exercer la profession d'architecte. Comme les nobles, les architectes s'efforcent de donner à leurs privilèges un statut héréditaire.

Dans l'élan fonctionnaliste issu de l'idéologie progressiste du XIX ${ }^{\mathrm{e}}$ siècle, les architectes du XX $\mathrm{XX}^{\mathrm{e}}$ siècle tentent une troisième fois d'œuvrer pour le développement de la démocratie. Ainsi, Le Corbusier (1925) énonce que tous les hommes ont les mêmes besoins : "Si nos esprits sont divers, nos squelettes sont semblables, nos muscles occupent la même place et réalisent les mêmes fonctions : dimensions et mécanismes sont donc déterminés." Malheureusement, Le Corbusier en vient en toute logique à la "machine à habiter", cellule idéale de l' "homme-machine" faisant partie de "villes-machines" dans lesquelles des hommes parfaitement conditionnés ont accès à un bonheur bien organisé ${ }^{9}$. Les grands ensembles modernistes au style internationalisé sont des expressions maladroites de ces grands principes, "des sortes de camps de dressage où l'on apprend la discipline aussi bien en circulant dans le maillage des lignes droites et orthogonales des rues [...] qu'en 'apprenant à habiter' des logements types, pour un homme moyen type" (Ragon, 1977). Les architectes fonctionnalistes cachent difficilement leur esprit tyrannique et les principes architecturaux qu'ils suivent ont été facilement intégrés dans la conception d'environnements construits en phase avec les périodes autoritaires du capitalisme, comme du socialisme. L'architecte moderniste se pense capable d'améliorer la vie de l'homme ordinaire grâce aux édifices qu'il projette. Mais il infantilise les habitants lorsqu'il prédétermine leurs relations avec l'environnement, pensant qu'ils vivront mieux s'ils prennent conscience de ce changement qualitatif de l'environnement. Ainsi, Le Corbusier ira jusqu'à affirmer que l'architecture moderniste doit être expliquée à l'opinion publique, il faut apprendre à celle-ci un "savoir habiter", le peuple doit être instruit de son devoir d' "habiter dignement".

Après Mai 1968, sous l'influence des luttes urbaines contre les rénovations brutales de quartiers historiques et d'initiatives des milieux marginaux contestataires (des architectures sans architectes, spontanées, autochtones, vernaculaires, indigènes, rurales, marginales, populaires, sans pedigree, anonymes...), l'architecte n'a plus le monopole du discours architectural. La parole est donnée prioritairement aux usagers/habitants. Après la faillite du modernisme, des architectes soutiennent la

\footnotetext{
9 Au XVII ${ }^{\mathrm{e}}$ siècle, parallèlement à l'émergence de la science moderne, les automates sont à la mode et une vision mécaniste du réel s'installe. René Descartes (1637) développe la thèse métaphysique controversée de l' "animal-machine" selon laquelle l'animal serait une machine dénuées de conscience et constituée par un assemblage de pièces et de rouages. Au XVIII ${ }^{\mathrm{e}}$ siècle, Julien Offray de La Mettrie (1748) radicalise ce point de vue et il propose la thèse de l' "homme-machine", selon laquelle l'homme lui-même serait assimilé à une machine. Derrière l'engouement pour les automates se cache le fantasme de la création de l'être vivant par l'homme lui-même sans l'aide d'une puissance divine extérieure.
} 
démocratie une quatrième fois en intégrant plusieurs dimensions nouvelles dans la conception des projets d'architecture : participation (phase libérale du capitalisme), autogestion (idéologie gauchiste), autoconstruction (faites votre maison vous-même, contre la division du travail), écologie (autodéplacement, autarcie alimentaire et vestimentaire). Mais l'émergence de ce type d'architecture nécessite l'invention des structures nécessaires à l'autogestion participative de l'habitat. De manière générale, une éducation générale à la culture architecturale dès le plus jeune âge doit être organisée et une information détaillée sur tous les projets d'urbanisme envisagés doit être donnée. Ce qui s'est révélé difficile à mettre en œuvre à grande échelle.

Dès les années 1970, selon Michel Ragon (1977), après avoir tout su, les architectes nous avouent qu'ils ne savent plus rien! L'image idéalisée de l'architecte s'étiole définitivement et le statut social de l'architecte ne cesse de se précariser. Avec le développement de la division du travail, la position de chef d'orchestre de l'architecte n'est plus tenable : les technocrates lui enlèvent le pouvoir de décision et ils imposent les conditions des appels d'offres publics, l'administration mise sur les bureaux d'études de toutes sortes, les services d'urbanisme lui imposent des règlementations complexes et contradictoires, l'administration du patrimoine fige des parties d'édifices, l'ordre des architectes lui impose des contraintes déontologiques désuètes, les promoteurs le placent dans un rôle d'exécutant au profit d'une stratégie marketing, les ingénieurs lui prennent le rôle d'innovateur technique, les consultants lui reprennent les études énergétiques, les informaticiens font du Building Information Modeling (BIM)... Tout au plus, l'architecte, dont la responsabilité civile sous surveillance juridique étroite n'est pas réduite pour autant, à l'autorisation de dessiner une belle façade...

Le pouvoir politique utilise également moins les architectes que par le passé pour affirmer son pouvoir. Dans notre société contemporaine, le flux rapide des informations remplace facilement la construction lente difficile et chère d'édifices pérennes. La durée des chantiers des grands projets architecturaux - opérations de prestige du pouvoir en place - est tellement longue que ces monuments sont dépassés avant d'être finis en engloutissant l'argent public. D'ailleurs, alors qu'il habite les réseaux sociaux, l'être humain trouve aujourd'hui une assise existentielle dans une condition assumée de "quasi-sujet" (Serre, 1983), située dans des "hyper-lieux" (Mongin, 2013) branchés sur le virtuel. Le succès de la démocratie associée à une communication effrénée a un effet pervers important: la dilution de la prise de décision entre un (trop) grand nombre d'acteurs (élus, collèges d'experts, comités de quartiers, investisseurs, associations, habitants...) empêche l'architecte d'exercer une synthèse architecturale. Comme l'écrit l'architecte Anne Démians (2013) : "Trop de concertations ont tué l'expressivité et la cohérence des projets".

Heureusement, l'architecte pourrait reconquérir un statut professionnel privilégié puisqu'il est repris en bonne place dans la liste des métiers non-menacés par l'automatisation du travail (Bakhshl, Benedikt Frey, Osborne, 2015). En effet, l'architecte serait plus difficilement remplaçable par un robot que d'autres, simplement parce qu'il utilise son imagination ou des idées originales pour créer des choses qui n'existent pas encore. Une compétence qui semble fort utile à l'avenir !

\section{COSMOS, COSMOGONIE ET DÉMIURGE}

En partant du principe que l'architecture n'est pas simplement la construction, les concepts d'architecture et de monde sont intimement liés. Comme le souligne l'historien allemand Tilo Schabert (1997), ces deux concepts "se réfléchissent l'un l'autre". D'un côté, dans la constructio mundi, le mot monde sous-entend le mot architecture. De l'autre, l'architecture "porte le déploiement du monde" en tant qu'espace organisé. D'ailleurs, le mot grec kósmos (ordre), devenu cosmos (monde) en latin, réunit les deux idées d'architecture et de monde. Le cosmos désigne pendant l'Antiquité toute figure bien ordonnée, la perception de l'expression d'un ordre dans lequel toutes les parties sont articulées dans un tout. Dans le monde des grecs, vu par eux comme un cosmos, toutes les parties s'intègrent à l'architecture d'un monde.

À cette conception du monde, correspond la représentation de l'architecte créateur de mondes : Platon écrit qu'un theos tektonikos (dieu démiurge) a édifié le monde dans le Timée (env. V ${ }^{\mathrm{e}} \mathrm{s}$. av. J.-C.) ; le Dieu créateur est l'architecte du temple et le bâtisseur de la ville sainte de Jérusalem dans l'Ancien 
Testament ; artifex est le dieu auquel saint Augustin s'adresse dans les Confessions (fin du IV ${ }^{\mathrm{e}} \mathrm{s}$.) ; Kant utilise le symbolisme de l'architecture pour représenter le mode d'édification de la pensée dans La critique de la faculté de juger $(1790)^{10}$; Claude Lévi-Strauss indique que toute civilisation humaine se réalise dans des formes obéissant à une structure inconsciente qu'il appelle une architecture logique dans son Anthropologie structurale (1958); d'après Robert Venturi (1966), même la formule célèbre de l'architecte américain Louis I. Kahn, "ce qu'une chose désire être", sous-entend implicitement son inverse "ce que l'architecte désire que devienne une chose". D'ailleurs, Schabert (1997) écrit que "la pensée humaine est cosmologique" par ses "qualités architecturales" et il poursuit en écrivant qu' "elle crée sa structure dans la structure des choses". En effet, penser en architecte, c'est construire dans le $R^{\prime}$ une interprétation du réel $R$, à partir de la perception répétée d'informations filtrées par les organes perceptifs. Cette construction (inter)subjective est structurée dans le $R^{\prime}$ du concepteur, puis projetée sur le $R$. L'architecte crée intentionnellement une nouvelle structure (un projet d'architecture parmi d'autres possibles) dans la structure sous-jacente du $R$ qu'il projette (celle qu'il révèle parmi d'autres possibles). Il projette donc une structure dans la structure des choses qu'il interprète.

Les mots architecture et monde mènent aux mots démiurge et cosmogonie.

Les cosmogonies sont des hypothèses métaphysiques, des grands récits et des mythes fondateurs idéaux et intemporels, qui expliquent l'origine du $R$ en puisant dans la richesse créative du $R^{\prime}$. Elles peuvent décrire l'origine du monde, mais également des dieux, des hommes, des choses... Le mot cosmogonie vient d'ailleurs des deux mots grecs kósmos (ordre), devenu cosmos (monde) en latin, et gon (engendrer). À l'inverse, les cosmologies sont des modèles scientifiques qui tentent d'expliquer l'origine et la nature du $R$, à partir de l'observation de faits et de lois physiques, construites par réduction dans le $R^{\prime}$. Le mot cosmologie venant des deux mots grecs kósmos et logos (raison, discours). Mais les cosmologies peuvent être des cosmogonies déguisées qui masquent les incertitudes des modèles scientifiques qui les sous-tendent.

Historiquement, la figure du démiurge est présente de manière continue dans toutes les cultures. L'universalité du thème vient de l'impuissance de l'être humain à connaître totalement le $R$. Le mot demiourgos (démiurge) vient de dêmos (gens du commun, peuple) et d'ergos (travail, artisan, fabricant). Le démiurge est donc littéralement le constructeur, le fabricateur du monde. La première mention du démiurge - dieu organisateur et créateur du monde, éternel géomètre - est souvent attribuée à Platon dans le Timée. Dans la bible, une occurrence du mot demiourgos est associée à dieu en tant que constructeur ${ }^{11}$. De nombreux auteurs latins et médiévaux opèrent un rapprochement entre les mots démiurge et architecte (Plaute, Cicéron, saint Augustin...). Au Moyen Âge, le chercheur français Louis Callebat (1999) affirme que l'architectus identifie le dieu créateur et ordonnateur du monde. Alors que l'expression biblique d'architectus sapiens est parfois associée à celle de Spiritus Sanctus (Esprit-Saint).

Le récit cosmogonique présente habituellement le $R$ comme une construction avant de la réduire à une fabrication. Le démiurge est justement l'être responsable qui fabrique l'univers (un créateur, une force, un dieu...). Dans les récits, il organise le $R$, il répartit les parties qui composent le tout pour les rendre belles et intelligibles, il donne un ordre idéal au monde (proportions, formes, sons, nombres...). Il a la capacité de créer le $R$ pour tous les autres.

$\mathrm{Au}$ commencement de la plupart des récits, selon le philosophe français Alain Delaunay (2016), le démiurge pose un geste fondateur, à partir duquel toutes les autres étapes d'émergence du monde vont se succéder (séparation des eaux d'en haut et des eaux d'en bas, séparation de la lumière et des ténèbres...). Ensuite, il pose une série de gestes secondaires. Quelques variantes existent telles que le travail de la matière organique en cuisine avec des ingrédients secrets, l'entretient du feu/souffle pour

\footnotetext{
10 "Toute science est en soi un système, et il ne suffit pas dans une science de construire selon des principes et de procéder ainsi techniquement, mais on doit au contraire y œuvrer architectoniquement comme dans un édifice existant pour luimême, et la traiter non pas comme une annexe ou une partie d'un autre édifice, mais comme un tout fonctionnant en soi, bien que l'on puisse ensuite aménager un passage de celui-ci à celle-là."

11 "Car il attendait la cité qui a de solides fondements, celle dont Dieu est l'architecte et le constructeur." Nouveau Testament, Hébreux, chapitre 11, verset 10.
} 
maîtriser les transformations de la matière ou forger des armes ultimes. Mais la majorité de ces gestes s'inspirent d'actions à l'œuvre pour architecturer le monde. Le démiurge maîtrise l'espace et le temps (parcellaire, arpentage, défrichage, bornage, déploiement, mesure, mise en ordre...), il construit/bâtit le monde en tant qu'architecte/artisan (maçon, tailleur de pierre, charpentier...) avec les outils des bâtisseurs (équerre et compas). Enfin, il lie des éléments à l'aide des techniques du tisserand (tissu, fil, métier, fuseau, ciseaux, aiguille, nœud...) pour construire le monde (filet, réseau, tapisserie, toile...). Ce qui renforce encore le lien étymologique entre politique et architecture à partir du mot latin textus (tissé, tissu, trame, texte).

Le philosophe français Paul Valéry (1921) donne la définition la plus connue de cet architecte démiurge : "Mais le constructeur que je fais maintenant paraître, trouve devant soi pour chaos et pour matière primitive, précisément l'ordre du monde que le Démiurge a tiré du désordre du début. La nature est formée et les éléments sont séparés ; mais quelque chose lui enjoint de considérer cette œuvre inachevée, et devant être remaniée et remise en mouvement, pour satisfaire plus spécialement à l'homme. Il prend pour origine de son acte le point même où le dieu s'est arrêté".

\section{LE MYTHE DE LA DÉMOCRATIE DIRECTE}

Le mot démocratie vient des mots grecs dêmos (peuple) et kratein (commander, exercice du pouvoir), littéralement c'est l'exercice du pouvoir par le peuple. La démocratie est régulièrement réduite à l'idée d'un pouvoir limité, d'un état de droit, d'une politique de l'équilibre ayant fonction de protéger la vie, les biens et la liberté des citoyens qui la composent. Ce qui permet facilement de considérer la démocratie comme un "idéal trahi" dès qu'un dysfonctionnement du système apparaît. Mais, selon le philosophe français Norbert Lenoir (2006), cette définition étymologique est simpliste. En effet, le développement de la démocratie est non-linéaire, puisqu'il trouve son origine dans la tension émulatrice entre le citoyen et le pouvoir. Surtout, elle est simpliste au moins pour trois raisons :

- le lieu de souveraineté diffère de celui de l'exercice réel du pouvoir. D'un côté, la démocratie fait de l'entièreté du peuple l'origine absolue de tout pouvoir (l'illusion de la démocratie directe), de l'autre, elle exclut du pouvoir une grande partie de ce même peuple par ses mécanismes représentatifs (l'illusion d'une représentativité objective) ;

- la nature du peuple censé exercer le pouvoir dans une démocratie est floue. Est-ce un peuple mythique, homogène et sans tensions, sans intérêts divergents et en permanence intéressé par la chose publique? Non, la démocratie est composée par un peuple hétérogène traversé par des intérêts divergents et contradictoires et elle repose sur l'art du consensus. La démocratie n'est pas un concept figé, elle est une dynamique émergente de tensions contradictoires ;

- le droit à la liberté individuelle est censée s'arrêter à celle des autres. En effet, chaque membre du système socioculturel a le droit d'être différent. Mais, en même temps, l'individualisme à outrance détruit la vie communautaire.

En démocratie, le citoyen et le pouvoir interagissent constamment. La liberté du citoyen ne veut pas dire qu'il échappe à la volonté d'autres personnes ou à des décisions institutionnelles. Chaque agent possède un potentiel variable d'actions en fonction d'un projet et ces actions/projets peuvent être incompatibles. Les institutions organisent les conditions de la liberté relative du citoyen, les conditions à partir desquelles chaque agens peut agir tout en garantissant l'intérêt public. De son côté, le citoyen interagit avec des décisions prises par d'autres en les contestant ou en changeant de représentants ce qui lui permet d'influencer la définition de cet intérêt public.

Du point de vue de l'environnement construit, le lieu de vie de l'entièreté du peuple ne fonde pas le pouvoir démocratique. L'architecture du pouvoir n'est pas l'architecture des gens ordinaires. Proposer une même architecture pour tous avec une visée démocratique, ne signifie pas pour autant que tous ont les mêmes droits à l'architecture ou que tous veulent nécessairement la même architecture. 


\section{CONCLUSIONS : LE FANTASME DU DÉMIURGE}

Confronté aux limites perceptives de la conscience, l'être humain développe pour agir une configuration augmentée, évolutive et opérationnelle du réel. Le décalage permanent entre les réactions parfois incompréhensibles du réel $R$ (incertitude) et le réel augmenté $R^{\prime}$ patiemment construit par l'être humain (pseudo-certitude) est à la source d'une angoisse existentielle latente.

Chacun possède un potentiel d'actions à mener en fonction d'un projet téléologique cherchant à réduire l'angoisse existentielle. Les incompatibilités potentielles de ces actions demandent une régulation en fonction d'un intérêt commun (encore faut-il un consensus sur la nature de cet intérêt commun). Pour permettre à chacun d'agir dans la vie de tous les jours, la gestion de ces relations est transmise à d'autres en leurs donnant la responsabilité de configurer le $R^{\prime}$ à notre place.

Parmi d'autres, deux processus permettent à certains de configurer le $R^{\prime}$ à la place d'autres : la politique et l'architecture. Des rapports étroits existent entre les systèmes politiques et l'architecture pour des raisons étymologique et sémantique, mais surtout parce que ces deux processus équilibrent, chacun à leur manière, les rapports dialogiques entre trois polarités: le système socioculturel, l'être (auto)organisé et l'environnement écosystémique. La politique conditionne l'environnement construit et l'architecture est un acte politique dans le sens où ils opèrent tous les deux un équilibre entre ces trois polarités, bien qu'ils agissent principalement sur des polarités différentes. En effet, la mise en place de systèmes politiques pour configurer le $R^{\prime}$ du peuple joue plutôt sur les interactions entre les membres du système socioculturel, alors que la projetation/fabrication d'artefacts architecturaux configure plutôt l'environnement écosystémique dans lequel habite ces membres.

Lorsqu'il gouverne, le politique est attiré par la figure du dirigeant qui guide. Alors que le mot dictateur vient de dictare (dicter), issu de dicere (dire), le mot diriger vient du mot latin dirigere, issu du verbe regere (guider). Dans un régime démocratique, un gouvernant ne devrait donc pas dicter une conduite, mais guider les gouvernés à adopter un comportement social. Bien que la gestion des interactions entre les membres du groupe socioculturel lui a été déléguée, le gouvernant, en tant qu'être humain, possède un potentiel d'action en fonction d'un projet téléologique. En tant que guide, il peut croire que ce projet personnel est utile pour l'intérêt de tous. Mais cette visée peut rapidement infantiliser les gouvernés dont les projets individuels sont étouffés par celui du gouvernant.

Le fantasme nostalgique de l'architecte démiurge s'inscrit dans une longue tradition commune étymologique et sémantique - entre architecture (configuration du $R$ en fonction d'un projet) et cosmogonie (explication de l'origine de la configuration du $R$ ). Le fantasme du démiurge dont souffre l'architecte est le reflet d'un désir (in)conscient de rendre l'être humain acteur du déploiement du $R$. Ce besoin impossible à réaliser persiste et l'agens tente sans cesse de dépasser la "bounded rationality" (Simon, 1957) qui l'affecte pour appréhender globalement le monde qui l'entoure. À la fois archaïque et contemporain, ce désir le pousse à expliquer l'origine et à maîtriser la situation actuelle du $R$.

Malheureusement, ce fantasme peut rapidement se transformer en hallucination. L'architecte conçoit alors des projets hors époque/contexte qui ne verront jamais le jour. Il peut aussi se transformer en chimère, lorsque l'architecte se rend compte qu'il n'a que le pouvoir d'imaginer, de potentialiser l'émergence de lieux de vies, puisque le politique décide pour lui de la réalisation (ou non) des projets. Lorsqu'il conçoit des projets d'architecture, l'architecte réalise des projets par substitution à répétition, et il peut finir par fantasmer que la vie est comme un projet.

La conscience des concepteurs de l'environnement construit - les architectes - est déchirée entre une attirance du pouvoir débridée (organiser l'environnement construit des autres) et une énorme empathie (mettre tout en œuvre pour faire émerger les envies d'organisations des autres). Cet état de conscience chronique vient de l'histoire mouvementée de la discipline, dans laquelle l'architecte n'a pas toujours eu le meilleur rôle. En effet, l'image de l'architecte a souvent été associée à celle de l'artiste chargé de l'édification d'un environnement construit capable d'affirmer la mise en place d'un pouvoir. Elle a été associée beaucoup plus rarement à celle du défenseur des droits des gouvernés.

La conscience de l'architecte est animée, d'un côté, par son aspiration à la "philosophie de l'étendue", qui sous-entend la connaissance distante et indirecte de l'espace, acquise rationnellement par 
l'utilisation de la métrique et, de l'autre côté, par la "philosophie de la centralité" qui donne à l'aide de la proxémique sa réalité à la spatialité par l'évidence sensible de la perception immédiate de l'individu, à partir de son moi, du centre de son monde, de son hic et nunc (Moles \& Rohmer, 1972). La philosophie de l'étendue qui organise à distance les interactions entre les membres du système socioculturel est nécessaire dans un système démocratique ayant un projet global d'évolution en fonction d'un but partagé. Mais elle peut être facilement détournée par le pouvoir pour répondre aux attentes d'une partie réduite de la population.

Au plus profond de la conscience des architectes, une "nostalgie du divin" est encore présente (Ragon, 1977), ce qui complique leurs rapports de fierté/honte avec le pouvoir. Cette situation est renforcée par la précarité actuelle de leur statut professionnel. Des jeux de pouvoir existent donc entre le gouvernant attiré par la figure du guide et l'architecte obsédé par le fantasme du démiurge. Le gouvernant et l'architecte ont horreur de l'incertitude !

\section{RÉFÉRENCES}

AUGUSTIN (saint). Confessions. [Trad. Mondadon, L. (de), Paris : Seuil (coll. Points), 1982.]

BAKHSHL, Hasan, BENEDIKT FREY, Carl, OSBORNE, Michael (2015). "Creativity versus robots: The creative economy and the future of employment". Rapport Nesta, avril 2015.

CALLEBAT, Louis (1999). "Architecte : histoire d'un mot". Voces, 10-11, 1999-2000, pp.47-58.

CLAEYS, Damien (2013). Architecture \& complexité : Un modèle systémique du processus de (co)conception qui vise l'architecture. Thèse de doctorat de l'Université catholique de Louvain. Louvain-la-Neuve : Presses universitaires de Louvain, 445pp.

COUFFIGNAL, Louis (1963). La cybernétique. Paris : PUF (coll. Que sais-je ?).

COULOUBARITSIS, Lambros (2014). La philosophie face à la question de la complexité : Le défi majeur du $X X I^{e}$ siècle. Tome 1 : Complexités intuitive, archaïque et historique / Tome 2 : Complexités scientifique et contemporaine. Bruxelles : Ousia.

DÉMIANS, Anne (2013). Carte blanche: "Les architectes et le(s) pouvoir(s)". Office et Culture, nov. 2013, $\mathrm{n}^{\circ} 30$.

DELAUNAY, Alain (2016). "Démiurge". Encyclopcedia Universalis. Site de l'Encyclopédie Universalis [en ligne], http://www.universalis-edu.com/encyclopedie/demiurge.

DESCARTES, René (1637). Discours de la méthode pour bien conduire sa raison et chercher la vérité dans les sciences. Paris : Hachette (coll. Poche), éd.2000.

DRAGAN, Radu (2008). "L'anthropologie du pouvoir dans l'architecture des régimes totalitaires". In IOSA, Ioana (dir.) (2008). L'architecture des régimes totalitaires face à la démocratisation. Paris: L'Harmattan (coll. Aujourd'hui l'Europe), pp.17-31.

FAREL, Alain (1991). Architecture et complexité : Le troisième labyrinthe. Marseille: Parenthèses (coll. Eupalinos), éd.2008.

FOUCAULT, Michel (1984). "Le sujet et le pouvoir". Trad. Durand-Bogaert, F. ["The subject and power"]. Dits et écrits. Paris : Gallimard, tome IV, éd.1994.

GROPIUS, Walter (1956). "Apollon dans la démocratie". Allocution prononcée à la réception du Hansische Goethepreis à Hambourg.

GUIBERT, Daniel (1987). Réalisme et architecture: L'imaginaire technique dans le projet moderne. Bruxelles/Liège : Mardaga (coll. Architecture + Recherche).

IOSA, Ioana (dir.) (2008). L'architecture des régimes totalitaires face à la démocratisation. Paris : L'Harmattan (coll. Aujourd'hui l'Europe).

JENCKS, Charles (1977). Le langage de l'architecture post-moderne. Trad. Collet C. \& Vallée Ch. (de) [The Language of Post-Modern Architecture. London: Academy Editions]. Paris : Denoël, éd.1984.

KANT, Emmanuel (1790). Critique de la faculté de juger. Trad. Alquié F. [Kritik der Urteilskraft]. Paris : Gallimard (coll. Folio/essais), éd.1989.

KUHN, Thomas (1962). La structure des révolutions scientifiques. Trad. Meyer L. [The Structure of Scientific Revolutions. Chicago: The University of Chicago Press]. Paris : Flammarion, éd.2008.

LATINI, Brunetto (1265). Li livres dou tresor. 
LE CORBUSIER (1925). L'Art décoratif d'aujourd'hui. [Paris: Crès (coll. L'Esprit nouveau)]. Paris : Flammarion (coll. Champs), éd.1996. [Rassemble les articles parus sans signature en 1924 dans L'Esprit nouveau.]

LÉVI-STRAUSS, Claude (1955). Tristes tropiques. Paris : Plon (coll. Terre Humaine), éd.2001.

LÉVI-STRAUSS, Claude (1958). Anthropologie structurale. Paris : Plon (coll. Agora), éd.2003.

LENOIR, Norbert (2006). La démocratie et son histoire. Paris : PUF (coll. L'interrogation philosophique).

LA METTRIE, Julien Offray (de) (1748). L'homme-machine. Paris : Gallimard (coll. Folio essais), éd.1999.

MARREY, Bernard (2013). Architecte : Du maître de l'œuvre au disagneur. Paris : Linteau.

MOLES, Abraham A. \& ROHMER, Élisabeth (1972). Psychologie de l'espace. Bruxelles : Casterman (coll. Mutations - Orientations).

MONGIN, Olivier (2013). La ville des flux : L'envers et l'endroit de la mondialisation urbaine. Paris : Fayard.

MORIN, Edgar (2001). La méthode 5 : L'humanité de l'humanité. L'identité humaine. Paris : Seuil (coll. Points).

NORBERG-SCHULZ, Christian (1974). La signification dans l'architecture occidentale. Trad. Dominicis A.M. (de) [Significato nell' architettura occidentale. Milano: Electa]. Liège/Bruxelles : Mardaga, éd.1977.

PLATON. Timée. [Trad. Brisson L., Paris : Flammarion (coll. Garnier Flammarion), éd.2001.]

PLATON. La République. [Trad. Leroux G., Paris : Flammarion (coll. Garnier Flammarion), éd.2004.]

RAGON, Michel (1977). L'Architecte, le Prince et la Démocratie : Vers une démocratisation de l'architecture? Paris : Albin Michel.

SCHABERT, Tilo (1997). L'architecture du monde: Une lecture cosmologique des formes architectoniques. Trad. Pierre Rusch [Die Architektur der Welt: Eine kosmologische Lekture architektonischer Formen. Munich: Wilhelm Fink]. Paris : Verdier (coll. Art et architecture), éd.2012.

SERRES Michel (1983). Rome : Le livre des fondations. Paris : Grasset.

SIMON, Herbert A. (1959). "Theories of Decision-making in Economics and Behavioral Science". The American Economic Review, vol. 49, n³, juin, pp.253-283.

SIMON, Herbert A. (1957). Models of Man Social and Rational: Mathematical Essays on Rational Human Behavior in a Social Setting. New York: Wiley.

TSIOMIS, Yannis (2008). "Architecture totalitaire ou discours totalitaires sur l'architecture ?". IOSA, Ioana (dir.) (2008). L'architecture des régimes totalitaires face à la démocratisation. Paris : L'Harmattan (coll. Aujourd'hui l'Europe), pp.32-41.

VALÉRY, Paul (1921). "Eupalinos ou l'Architecte". Valéry, Paul (1960). Euvres II. Paris : Gallimard (coll. Pléiade), éd.2008, pp.79-147.

VENTURI, Robert (1966). De l'ambiguïté en architecture. Trad. Schlumberger M. \& Vénard J.-L. [Complexity and contradiction in Architecture. New York: The Museum of Modern Art]. Paris : Dunod, éd.1999.

VITRUVE (env. -25 av. J.-C.). Les dix livres d'architecture. [Trad. Perrault, C., De Architectura (1673). Liège/Bruxelles : Mardaga, éd.1979.]

WEBER, Max (1905). L'Éthique protestante et l'esprit du capitalisme. [Die protestantische Ethik und der 'Geist' des Kapitalismus]. Paris : Plon (coll. Agora), éd.1985.

WIENER, Norbert (1948). Cybernetics, or Control and Communication in the Animal and the Machine. Cambridge: MIT Press. 